\title{
THE CERN AXION SOLAR TELESCOPE (CAST): STATUS AND PROSPECTS
}

\author{
I. G. IRASTORZA ${ }^{a *}$ S. ANDRIAMONJE ${ }^{b}$, E. ARIK ${ }^{c}$, D. AUTIERO ${ }^{a}$,

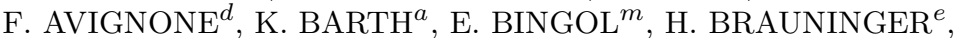

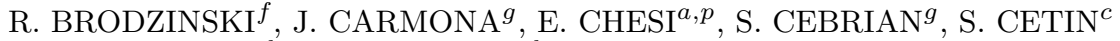

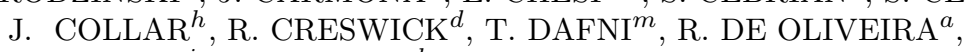 \\ S. DEDOUSSIS ${ }^{i}$, A. DELBART ${ }^{b}$, L. DI LELLA ${ }^{a}$, C. ELEFTHERIADIS ${ }^{i}$, \\ G. FANOURAKIS ${ }^{j}$, H. FARACH ${ }^{d}$, H. FISCHER ${ }^{k}$, F. FORMENTI ${ }^{a}$, \\ T. GERALIS ${ }^{j}$, I. GIOMATARIS ${ }^{b}$, S. GNINENKO ${ }^{l}$, N. GOLOUBEV ${ }^{l}$, \\ R. HARTMANN ${ }^{e}$, M. HASINOFF $^{a, p}$, D. HOFFMANN ${ }^{m}$, J. JACOBY ${ }^{m}$, \\ D. $\mathrm{KANG}^{k}$, K. KÖNIGSMANN ${ }^{k}$, R. KOTTHAUS ${ }^{n}, \mathrm{M}^{2} \mathrm{KRCMAR}^{o}$, \\ M. KUSTER ${ }^{e}$, B. LAKIC ${ }^{o}$, A. LIOLIOS ${ }^{i}$, A. LJUBICIC ${ }^{o},{\mathrm{G} . \mathrm{LUTZ}^{n}}^{n}$,

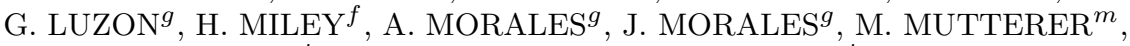 \\ A. NIKOLAIDIS ${ }^{i}$, A. ORTIZ $^{g}$, T. PAPAEVANGELOU ${ }^{i}$, A. PLACCI $^{a}$, \\ G. RAFFELT ${ }^{n}$, H. RIEGE ${ }^{a}$, M. SARSA ${ }^{g}$, I. SAVVIDIS ${ }^{i}$, R. SCHOPPER $^{m}$, \\ I. SEMERTZIDIS ${ }^{m}$, C. SPANO ${ }^{k}$, J. VILLAR $^{g}$, B. VULLIERME ${ }^{a}$, \\ L. WALCKIERS ${ }^{a}$, K. ZACHARIADOU ${ }^{j}$, K. ZIOUTAS ${ }^{a, i}$ \\ ${ }^{a}$ European Organization for Nuclear Research (CERN), Geneve, Switzerland \\ ${ }^{b}$ DAPNIA, Centre d'Etudes de Saclay (CEA-Saclay), Gif-Sur-Yvette, France \\ ${ }^{c}$ Department of Physics, Bogazici University, Istambul, Turkey \\ ${ }^{d}$ Department of Physics and Astronomy, U. South Carolina, Columbia, Sc, USA \\ ${ }^{e}$ Max-Planck-Institut für Extraterrestrische Physik, MPG, Garching, Germany \\ ${ }^{f}$ Pacific Northwest National Laboratory, Richland, Wa, USA \\ ${ }^{g}$ Instituto de Física Nuclear y Altas Energías, Universidad de Zaragoza, Zaragoza, Spain \\ ${ }^{h}$ Enrico Fermi Institute, University of Chicago, Chicago, Il, USA \\ ${ }^{i}$ Aristotle University of Thessaloniki, Thessaloniki, Greece \\ ${ }^{j}$ National Center for Scientific Research "Demokritos" (NRCPS), Athens, Greece \\ ${ }^{k}$ Albert-Ludwigs-Universität Freiburg, Freiburg, Germany \\ ${ }^{l}$ Institute for Nuclear Research (INR), Russian Academy of Sciences, Moscow, Russia \\ ${ }^{m}$ Institut für Kernphysik, Technische Universitat Darmstadt, Darmstadt, Germany \\ ${ }^{n}$ Max-Planck-Institut für Physik, Munich, Germany \\ ${ }^{\circ}$ Ruder Boskovic Institute, Zagreb, Croatia \\ ${ }^{p}$ Department of Physics and Astronomy, U. of British Columbia, Vancouver, Canada
}

The CAST experiment is being mounted at CERN. It will make use of a decommissioned LHC test magnet to look for solar axions through its conversion into photons inside the magnetic field. The magnet has a field of 9 Tesla and length of $10 \mathrm{~m}$ and is installed in a platform which allows to move it $\pm 8^{\circ}$ vertically and $\pm 40^{\circ}$ horizontally. According to these numbers we expect a sensitivity in axion-photon coupling $g_{a \gamma \gamma} \lesssim 5 \times 10^{-11} \mathrm{GeV}^{-1}$ for $m_{a} \lesssim 10^{-2} \mathrm{eV}$, and with a gas filled tube $g_{a \gamma \gamma} \lesssim 10^{-10} \mathrm{GeV}^{-1}$ for $m_{a} \lesssim 2 \mathrm{eV}$.

*attending speaker, e-mail: igor.irastorza@cern.ch 


\section{Introduction}

Axions are light pseudoscalar particles that arise in theories in which the Peccei-Quinn U(1) symmetry has been introduced to solve the strong CP problem $^{1,2}$. They could have been produced in early stages of the Universe being attractive candidates to the cold Dark Matter (and in some particular scenarios to the hot Dark Matter) responsible to the $1 / 3$ of the ingredients of a flat universe. Axions could also be copiously produced in the core of the stars by means of the Primakoff conversion of the plasma photons. In particular, a nearby and powerful source of stellar axions would be the Sun.

The solar axion flux can be easily estimated ${ }^{3,4}$ within the standard solar model, resulting in an axion flux of an average energy of about $4 \mathrm{keV}$. These solar axions (as well as cosmological ones) have been searched for in the past by a number of experiments ${ }^{6}$. In particular, there have been two previous experiments using magnets to search for them ${ }^{9,10}$, following the original idea of ${ }^{11}$. The CAST collaboration ${ }^{12}$ is using a decommissioned LHC test magnet which improves substantially the sensitivity to solar axions with respect to the previous experiments. In the following we make a short description of the experiment as well as its status and prospects.

\section{Description and status of the experiment}

As stated before, CAST uses a decommissioned LHC test magnet of 9 Tesla and $10 \mathrm{~m}$ long. The magnet is twin-aperture and has a effective cross section of $\sim 2 \times 14 \mathrm{~cm}^{2}$. At both ends, several detectors will look for the $\mathrm{X}$-rays originated by the conversion of the axions inside the magnet when it is pointing to the Sun. The magnet is mounted on a platform which allows a movement of $\pm 8^{\circ}$ vertically and $\pm 40^{\circ}$ horizontally. With this platform it can be oriented to certain directions and track the Sun during about three hours per day in average (1.5 at sunrise and 1.5 at sunset). The limitation in the movement of the magnet -and therefore in the amount of exposition to the Sun- is fixed by the cryogenic system that keeps it superconducting. In Fig. 1 an schematic view of the experimental setup is plotted, showing the magnet and the platform to move it. The position of the X-ray detectors at both ends of the magnet is also marked. At the time of writing this text, the full cryogenic installation is finished, the magnet reaching routinely its nominal current and magnetic field. The hardware and software of the tracking system are also fully operational, and the orientation of the magnet has been correlated with celestial coordinates.

The probability that an axion going through the transverse magnetic 


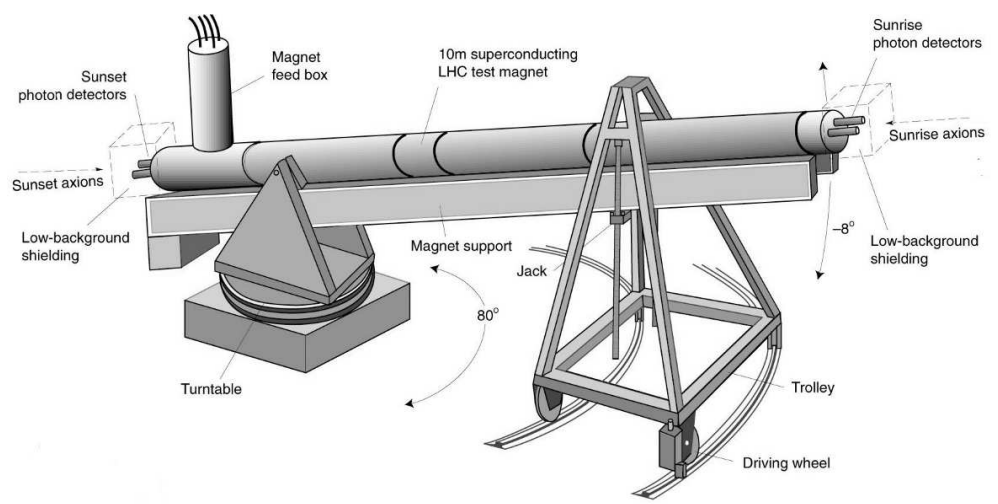

Figure 1. Schematic view of the CAST experimental setup. The $10 \mathrm{~m}$ long LHC test magnet is mounted on a platform like the one shown in the drawing, allowing a movement of $\pm 8^{\circ}$ vertically and $\pm 40^{\circ}$ horizontally. The detectors will be located at both ends of the magnet, and will be exposed of axion-induced X-rays during 3 hours per day in average.

field $B$ over a length $L$ will convert to a photon is given by:

$$
P_{a \gamma}=2.1 \times 10^{-17}\left(\frac{B}{9 \mathrm{~T}}\right)^{2}\left(\frac{L}{10 \mathrm{~m}}\right)^{2}\left(g_{a \gamma \gamma} \times 10^{10} \mathrm{GeV}^{-1}\right)^{2}|\mathcal{M}|^{2}
$$

where the matrix element $|\mathcal{M}|^{2}=2(1-\cos q L) /(q L)^{2}$ accounts for the coherence of the process, being $q$ the momentum exchange. In our case, the axion and photon waves keep in phase $\left(|\mathcal{M}|^{2} \simeq 1\right)$ for axion masses up to $\sim 10^{-2} \mathrm{eV}$, while for higher masses $|\mathcal{M}|$ begins to decrease, an so does the sensitivity of the experiment. To cope with this coherence loss, a second phase of CAST is planned filling the beam pipe inside the magnet with a gas to give a mass to the photons. For axion masses that match the photon mass, the coherence is restored. By changing the pressure of the gas inside the pipe the sensitivity of the experiment can be extended to higher axion masses.

Three different types of detectors have been developed to be used to detect the $\mathrm{X}$-rays originated by the conversion of the axions inside the magnet: a time projection chamber (TPC), a CCD and a micromegas detector. The TPC will profit of the stability and robustness of a well-known technique with position sensitivity to single out axions coming out of the magnet bores. It is placed in one of the ends of the magnet looking for X-rays from "sunset" axions. In the other side of the magnet, facing "sunrise" axions, two different detectors will be working at the same time (recall that LHC magnets are twin aperture). One smaller gas chamber will test the more 
novel micromegas principle ${ }^{13}$ with a potentially higher rejection factor due to the better spatial resolution. A CCD detector or a smaller micromegas chamber will work in conjunction with a mirror system to focus X-rays coming out of the magnet bores. This special device is commented later on.

The installation of the detectors in the experimental site is in an advanced phase. Two of them (the TPC and the micromegas) are already installed on the corresponding ends of the magnet, and their normal operation has been checked. In fact, at the time of writing this text, some first preliminary hours of data have been taken with the TPC in "axionsensitive" conditions, i. e., with the magnet on and tracking the Sun. The installation phase of CAST will be fully completed when the third detector together with the focusing mirror system will be installed in the next days.

To obtain a reasonable background level several low background techniques are being used. The radioactivity of the components near the detectors have been measured, in order to avoid the presence of substantial background sources. CAST experimental site is at surface level, so the presence of cosmic rays will be an important component of the background. To cope with this, some shielding is being designed. In spite of weight and space constrains imposed by the experimental area and set-up, some preliminary tests with the TPC in the laboratory indicate that the background could be shielded down by one order of magnitude. Also a good part of the background can be rejected off-line due to the different signatures that $\mathrm{X}$-rays and most of the background leave in the detectors. In addition, the use of the above mentioned X-ray focusing mirror system is meant to further improve the signal to noise ratio and so the sensitivity of the experiment. Such a device, similar to those used in X-ray astronomy, will be able to focus axion induced X-rays from the magnet bore to a submillimeter spot. This will allow us to use a very small detector (with lower expected background) and increase the expected signal to background ratio by about two orders of magnitude.

The background optimization being still in progress, some preliminary test with the detectors show background levels below $10^{-5}$ counts $/ \mathrm{keV} / \mathrm{cm}^{2} / \mathrm{s}$ in the energy region of the solar axion spectrum, which is already very close to the objective of the proposal ${ }^{12}$, which foresee a $3-\sigma$ limit of $g_{a \gamma \gamma} \lesssim 5 \times 10^{-11} \mathrm{GeV}^{-1}$ in the axion mass range where coherence is preserved.

In Fig. 2 the complete expected exclusion plot is shown, including both phases of data taking: one year in vacuum and two more years with the 


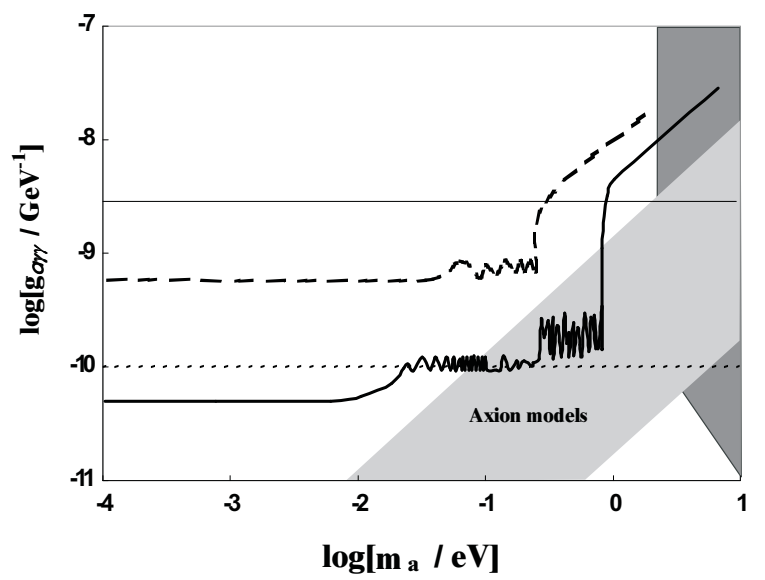

Figure 2. Expected exclusion limit of CAST (solid line) taking into account both data taking phases as explained in the text. Also shown are the limits obtained by SOLAX $^{7}$ and $\operatorname{COSME}^{8}$ experiments using solid state detectors (thin line), and the Tokyo "helioscope"10 (dashed line) using the same principle as CAST. The region favoured by theoretical axion models is also shown. Finally, the dotted line represents the theoretical red giant bound ${ }^{15}$ and the grey region on the right is excluded from the absence of an axion-decay quasi-monochromatic photon line from galactic clusters ${ }^{16}$.

tube filled with gas at varying pressure. The first phase is responsible of the low axion mass part of the limit $\left(m_{a} \lesssim 0.02 \mathrm{eV}\right)$, while the second phase will extend the limit to higher axion masses roughly to $g_{a \gamma \gamma} \lesssim 10^{-10}$ $\mathrm{GeV}^{-1}$ for $m_{a} \lesssim 1 \mathrm{eV}$. From Fig. 2 one can see that CAST will be able to improve substantially previous laboratory limits. In addition, it will go beyond some theoretical bounds like the red giant limit. It can also be seen that, during the second phase of data taking, CAST will be able to enter into some theoretically favored region of parameter space.

Finally, let's mention briefly some others goals of CAST that can be considered as by-products of the experiment. For instance, CAST could be able to detect the suggested monochromatic $14.4 \mathrm{keV}$ nuclear axion emission from the Sun's core ${ }^{14}$. Other kinds of axion emission have been proposed in more specific theoretical models, like Kaluza-Klein axion models, or the case where the axion is coupled to the electron, giving rise to axion-bremstrahlung emission. In addition, during the non-alignment periods there will be opportunities to observe other possible sources of axions in the sky. CAST could make some kind of "axion astronomy" by mapping the data taken during these periods on galactic coordinates. Examples of other possible axion sources are the galactic center, pulsars, GRBs and supernovae. 


\section{Conclusions}

The CAST experiment is now finishing its installation phase at CERN. The 9 Tesla and $10 \mathrm{~m}$ long LHC test magnet is mounted in a platform which allows to move it $\pm 8^{\circ}$ vertically and $\pm 40^{\circ}$ horizontally. The cryogenics of the magnet and the tracking system are ready and two gas detectors (a TPC and a micromegas) are already installed at both ends of the magnet. Some preliminary data have already been taken, and the definitive data taking phase will start as soon as the third detector and the X-ray focusing mirror system will be installed. Regarding the main purpose of the experiment, the search for "standard" hadronic solar axions, the preliminary tests allow us to keep the original goal of a sensitivity to axion-photon coupling down to $g_{a \gamma \gamma} \lesssim 5 \times 10^{-11} \mathrm{GeV}^{-1}$ for $m_{a} \lesssim 0.02 \mathrm{eV}$. A second phase of acquisition with a gas filled tube to provide a mass to the photons will allow to extend a limit $g_{a \gamma \gamma} \lesssim 10^{-10} \mathrm{GeV}^{-1}$ for axion masses up to $m_{a} \lesssim 1 \mathrm{eV}$. Some by-products of the experiment are foreseen, like the search for other model-

specific solar axion emissions, or the exploration of other axion sources in the sky.

\section{References}

1. R. D. Peccei and H. R. Quinn, Phys. Rev. Lett. 38 (1977) 1440.

2. G. Raffelt, Phys. Rep. 198 (1990) 1.

3. K. van Bibber, P. M. McIntyre, D. E. Morris and G. G. Raffelt, Phys. Rev. D 39 (1989) 2089.

4. R. J. Creswick, F. T. Avignone, H. A. Farach, J. I. Collar, A. O. Gattone, S. Nussinov and K. Zioutas, Phys. Lett. B 427 (1998) 235 [hep-ph/9708210].

5. E. Masso and R. Toldra, Phys. Rev. D 52 (1995) 1755 [hep-ph/9503293].

6. L. J. Rosenberg and K. A. van Bibber, Phys. Rept. 325 (2000) 1.

7. F. T. Avignone et al. [SOLAX Collaboration], Phys. Rev. Lett. 81 (1998) 5068

8. A. Morales et al. [COSME Collaboration], Astropart. Phys. 16 (2002) 325

9. D. M. Lazarus et al. Phys. Rev. Lett. 69 (1992) 2333.

10. Y. Inoue et al. Phys. Lett. B 536 (2002) 18 [arXiv:astro-ph/0204388].

11. P. Sikivie, Phys. Rev. Lett. 51 (1983) 1415 [Erratum-ibid. 52 (1984) 695].

12. K. Zioutas et al., Nucl. Instrum. Meth. A 425 (1999) 482 [arXiv:astro-ph/9801176].

13. Y. Giomataris, P. Rebourgeard, J. P. Robert and G. Charpak, Nucl. Instrum. Meth. A 376 (1996) 29.

14. S. Moriyama, Phys. Rev. Lett. 75 (1995) 3222 [arXiv:hep-ph/9504318].

15. G. G. Raffelt, Phys. Rev. D 33 (1986) 897; G. G. Raffelt and D. S. Dearborn, Phys. Rev. D 36 (1987) 2211.

16. M. T. Ressell, Phys. Rev. D 44 (1991) 3001; M. A. Bershady, M. T. Ressell and M. S. Turner, Phys. Rev. Lett. 66 (1991) 1398. 